\title{
Gradhiva
}

GRADHIV

Revue d'anthropologie et d'histoire des arts

$10 \mid 2009$

Présence africaine

\section{Entretien avec le poète, romancier et essayiste Daniel Maximin}

\section{(2) OpenEdition \\ Journals}

Édition électronique

URL : http://journals.openedition.org/gradhiva/1573

DOI : $10.4000 /$ gradhiva. 1573

ISSN : 1760-849X

Éditeur

Musée du quai Branly Jacques Chirac

Édition imprimée

Date de publication : 4 novembre 2009

Pagination : 156-163

ISBN : 978-2-35744-012-8

ISSN : 0764-8928

Référence électronique

"Entretien avec le poète, romancier et essayiste Daniel Maximin », Gradhiva [En ligne], 10 | 2009, mis en ligne le 03 février 2010, consulté le 01 mai 2019. URL : http://journals.openedition.org/ gradhiva/1573; DOI : 10.4000/gradhiva.1573

(C) musée du quai Branly 


\section{Entretien avec le poète, romancier et essayiste Daniel Maximin*}

\section{Quelle a été votre expérience avec Présence Africaine?}

Après la mort d'Alioune Diop, j’ai été chargé, avec Mukala Kadima, de m'occuper de la revue et de quelques-unes des collections des éditions, notamment la collection de poésie. Mais mon histoire avec Présence Africaine a commencé après le bac, au moment où j'ai entrepris, à l'âge de dix-sept ans, des études de lettres à la Sorbonne.

Depuis la Sorbonne, pour rejoindre la librairie de la rue des Écoles, il fallait descendre une rue en pente, une pente en quelque sorte naturelle pour un jeune homme du tiers-monde, un jeune Antillais qui avait soif, à travers tout ce qu'il avait lu, de découvrir le monde réuni autour de Présence Africaine.

On arrivait alors dans ce qu'on appelait «la Sorbonne des Noirs", ou encore "la Sorbonne du tiers-monde ». On entrait, au 25 bis rue des Écoles, dans une librairie qui n'avait rien d'intimidant, où il y avait des gens debout en train de discuter, des aînés comme des jeunes. Les aînés étaient Aimé Césaire, Léon-Gontran Damas, Léopold Senghor, Cheikh Anta Diop, Sembène Ousmane, Edouard Maunick, parmi tant d'autres de la génération suivante, avec au beau milieu d'eux la figure tutélaire du grand médiateur : Alioune Diop. Il y avait aussi son épouse, Christiane Diop, qui nous recevait comme une mère accueille ses enfants. D'ailleurs, elle disait «mes enfants » à tous les étudiants de passage. Il régnait une telle chaleur humaine dans cette librairie que nous nous y sentions chez nous, et pas dans un antre de grands hommes. Présence Africaine, c'était la maison, au sens d'un lieu dont la porte est constamment ouverte. Toute la génération des jeunes issus de la décolonisation se retrouvait dans cet endroit, à côtoyer les pères de la décolonisation!

Il y avait aussi, au 16 rue des Écoles, les bureaux d'Alioune Diop. Parfois, nous descendions discuter avec Césaire, Damas ou Alioune Diop, lequel avait une capacité d'écoute exceptionnelle et s'efforçait systématiquement de pénétrer la pensée d'autrui. Il suffisait qu'un jeune homme en année de maîtrise entre dans la librairie pour que Diop lui dise : «Racontez-moi. » De la même manière, Damas et Césaire demandaient à tous les jeunes qui passaient ce qu'ils étaient en train d'écrire. Je me souviens d'une jeune poétesse brésilienne qui était venue dans l'intention de commencer par lire ses propres poèmes. Il faut imaginer ce que cela représentait, pour des jeunes gens débutants, d'entendre de grands poètes leur demander de lire leur travail et de repartir avec ce genre de dédicace : «J'attends de vous que vous fassiez votre part. » Personnellement, j'ai des dédicaces de Damas, de Senghor et de Césaire qui disent toutes la même chose : "Jeune homme, nous comptons sur vous ", et non pas : "Merci de nous admirer.» C'était une véritable fraternité. C'est important de le préciser, car dans tout cela il n'y avait pas beaucoup d'idéologie à une époque où l'idéologie était pourtant omniprésente, avec le marxisme, le communisme, le socialisme, les thèses de Senghor, celles de Sékou Touré, etc. On aurait pu être tenté de choisir parmi tous ceuxlà, mais on préférait penser librement, c'est-à-dire écouter et discuter. Ce qui a beaucoup frappé ceux de ma génération qui ont connu Présence à ce moment-là, c'est l'extraordinaire qualité d'écoute qui s'y manifestait, notamment envers les jeunes. On peut dire que nous devons notre formation au fait d'avoir côtoyé un certain nombre de ces écrivains. 


\section{Quand vous dites «nous », de qui parlez-vous?}

Des étudiants qui passaient à la librairie, de tous ceux qui voulaient s'y arrêter : des Africains, des Antillais, des Brésiliens, des Noirs américains. Il n'y avait pas à Paris - il n'y en a toujours pas, d'ailleurs - de centre culturel francophone, ni du tiers-monde, ni des Antilles, ni de l'Afrique. Mais il y avait cet endroit à propos duquel on disait : «On passe à Présence. » Présence était en quelque sorte notre centre culturel. Il y avait des affiches, des papiers collés au mur qui annonçaient des pièces de théâtre, des conférences... La librairie était un véritable lieu d'informations culturelles, mais aussi politiques. Quand par exemple il y avait un coup d'État en Afrique, on allait à Présence. Sinon, où serions-nous allés nous informer?

\section{Quelles étaient les autres particularités de Présence Africaine ? C'était un lieu de discussion, une possibilité de prendre la parole?}

Le plus extraordinaire, et qui fait que cette maison a eu une importance absolument centrale dans l'histoire, c'est qu'elle était un lieu de rencontres, un lieu de passage, au vrai sens du terme. Il n'y avait ni réunion, ni colloque, ni conclave, le principe était : «Qui veut entre." Et une fois entré, on pouvait dialoguer avec les gens qui se trouvaient là. Avec Damas, par exemple, ou avec d'autres au comptoir du café d'en face. On réfléchissait sur les littératures, sur la culture, sur les indépendances, sur le destin de l'Afrique et du tiersmonde en général.

C'est parce qu'il favorisait un tel bouillonnement que ce lieu a rendu possible une chose très difficile : la rencontre collective entre les générations.

Par ailleurs, Présence n'avait rien d'une école. Quand on y allait, ce n'était pas comme quand on allait aux cours de la Sorbonne. On allait dans un lieu de questionnement, de rencontres et de dialogue. Car ce qui était très frappant, dans l'ensemble de cette génération, chez Alioune Diop, Cheikh Anta Diop, Césaire, Damas, Senghor et quelques autres, c'était la capacité de dialogue avec d'autres générations. Dans la mesure où ils entretenaient eux-mêmes un dialogue et une discussion sur la politique, la littérature et l'histoire du monde, ils n'avaient pas besoin de se présenter comme des pères qui veulent imposer aux jeunes ce qu'ils sont.

Quant à nous, on était là, on écoutait, on dialoguait. À partir de là, des affections et des amitiés se sont formées. Cela a été mon cas avec Alioune Diop, avec $M^{\text {me }}$ Diop, avec Césaire et Damas, principalement. Et aussi avec d'autres figures de la maison, par exemple Jacques Howlett, qui a joué un rôle si important auprès d'Alioune, ou Kala Lobé, qui s'occupait plus précisément de la revue.

L'esprit était donc à la fois familial et égalitaire, ce qui n'empêche pas que chacun était encouragé à faire son chemin tout seul. Et le fait de rencontrer tous ces gens ensemble, loin de créer un mouvement commun, favorisait au contraire l'individualité : Cheikh Anta avec ses idées sur l'histoire de l'Afrique, Césaire avec ses engagements poétiques et politiques, Damas qu'on appelait le «commis voyageur de la poésie " parce qu'il passait son temps à parcourir le monde pour trouver des anthologies. Il a publié la première anthologie en 1947, celle du Seuil, Poètes d'expression française, et à Présence Africaine, en 1966, l'énorme somme qui s'appelait Nouvelle somme de poésie du monde noir. Dans tous les cas, à Présence, on s'efforçait de publier tous les styles, toutes les générations. Présence cherchait aussi à réintroduire dans le dialogue du monde des gens qui en avaient été écartés. Ce n'était pas uniquement pour donner la parole à une partie du monde, mais pour dire que le monde a besoin de dialogue, particulièrement les oubliés, les Africains, les gens du tiers-monde, les gens de la Caraïbe ou les Noirs américains.

L'idée de base, c'était qu'il fallait donner la parole à ceux qui ne l'ont pas eue. Et cela dans tous les domaines, d'où l'importance du culturel. On s'est parfois demandé si Présence Afri- 
caine était une structure politique cachée. La vérité, c'est qu'au centre de l'action d'Alioune Diop (il avait été sénateur à un moment, il aurait pu continuer à faire de la politique), il y avait la conviction que le culturel doit avoir la primauté dans l'identité des peuples, le politique et les frontières venant par surcroît. Pour Alioune Diop, le culturel (la littérature, la poésie...) ne doit jamais être au service de l'action politique. C'est l'action politique qui doit être au service de l'émancipation culturelle. Alioune Diop a même inventé une expression pour définir ce qu'étaient les membres de Présence Africaine, il disait : « Nous sommes des hommes de culture. » Il préférait cette appellation à celle d'intellectuel, trop restrictive à ses yeux par rapport à l'imaginaire. La formule « homme de culture » incluait les musiciens, les plasticiens, les penseurs. Lui qui n'était ni créateur ni romancier, il a toujours accordé une place primordiale à l'expression par l'imaginaire en tant que métaphore de l'identité et des questions soulevées par l'histoire des peuples dont il parlait.

En ce sens, l'idée d'un lieu qui était en même temps une librairie et un lieu de rencontres était absolument primordiale. Césaire dit ainsi dans le Cahier d'un retour au pays natal: "Ma bouche sera la bouche des malheurs qui n'ont point de bouche. " Eh bien, les gens de Présence mettaient fin à des siècles de silence sur le tiers-monde et ouvraient leurs bouches sur les erreurs, sur ce que Senghor appelle "les rires Banania ", les aliénations, les «Tintin au Congo ", les images des individus opprimés, dominés et silencieux.

\section{D'autres revues françaises se sont engagées contre le colonialisme et ont publié des auteurs de Présence : Esprit, Les Temps modernes, Europe, Les Lettres nouvelles...}

C'est le même mouvement, effectivement, et il faut rappeler le lien qui existait entre toutes ces revues. On oublie souvent que Présence est un mouvement qui a commencé avec les chrétiens de gauche. Alioune Diop avait des liens étroits avec l'équipe du Seuil. Au début, c'est d'ailleurs au Seuil qu'il était hébergé, 27 rue Jacob. Ensuite, il a pris son autonomie en louant des bureaux rue Descartes, avant de s'installer rue des Écoles.

Avant et pendant la guerre, Senghor n'a cessé de dialoguer avec ses amis chrétiens de gauche, parmi lesquels les futurs fondateurs du Seuil. C'est ainsi que le Seuil a commencé son activité en publiant des auteurs du tiers-monde, comme Senghor avec Hostie noire, ou Damas avec son anthologie de 1947 qui rassemblait plus de soixante-dix écrivains allant de l'Indochine à la Guyane en passant par Haïti et les Antilles. Le lien avec Les Temps modernes était lui aussi très fort. L'exposition que la BNF a consacrée à Sartre a montré que pendant la guerre Alioune Diop et d'autres étudiants africains l'invitaient régulièrement au Foyer colonial, leur cercle culturel parisien, pour discuter de divers sujets, comme par exemple l'amour.

Présence Africaine a ainsi fait le lien entre différents mouvements culturels, littéraires ou idéologiques français souvent très opposés : les communistes, Les Temps modernes, les chrétiens de gauche d'Esprit et Camus. D'ailleurs, le comité de patronage du premier numéro de Présence Africaine doit être le seul endroit à avoir pu réunir, à l'époque, des individus aux opinions politiques si différentes.

L'une des grandes forces de Présence, c'est que c'était un lieu qui était ouvert à tous, Noirs et Blancs, Africains et Francais, unis dans le combat anticolonialiste. À cet égard, il n'est pas anodin que Georges Balandier ait été un temps rédacteur en chef de la revue. Il y avait aussi le philosophe Jacques Howlett, ou Michel Leiris... Même si le mouvement était mené par des Noirs, la rencontre se faisait au-delà de considérations raciales et ethniques.

\section{Pouvez-vous nous expliquer le combat d'Alioune Diop, qui était à la fois culturel et politique?}

La culture n'était pas perçue comme un domaine à part, indépendant de la politique. Au contraire, elle sous-tendait le combat politique. Présence Africaine faisait de la politique 
sans sectarisme, sans défendre une thèse plutôt qu'une autre. Alors que le grand ami de la revue était Senghor, elle n'en publiait pas moins les textes de Sékou Touré, qui ne suivait pourtant pas la même ligne. En ce sens, la revue était un outil au service de l'histoire. On n'y considérait pas le culturel comme hors du monde, mais comme l'aboutissement premier du politique. L'important, c'était l'émancipation, toutes les émancipations, quelles que soient les voix politiques qui s'exprimaient, parfois d'ailleurs dans des directions très divergentes... Présence était à l'écoute de ces voix et leur donnait la parole. Et ces idées circulaient entre des gens qui partageaient une même solidarité, une solidarité sans laquelle rien n'aurait été possible. L'idée commune consistait à dire : nous sommes libres et nous allons nous battre en tant que tels pour la libération politique. Or la culture était la preuve de cette liberté. Présence vient directement de là.

Présence Africaine n'était pas une revue idéologique. C'était une revue de grande ouverture au dialogue des cultures. Par exemple, Présence a passé pratiquement dix ans à débattre des conditions d'une littérature nationale. Ce débat a impliqué des jeunes et des anciens. Pendant que les auteurs de Présence se demandaient comment on allait faire des romans quand on serait libres politiquement, les romans se faisaient. Finalement, les auteurs étaient déjà en train de créer un patrimoine littéraire et poétique qui anticipait l'avènement d'une littérature francophone une fois que la libération politique serait acquise. L'autre débat était linguistique : quelle langue allons-nous employer à la libération? le wolof, le bambara, le swahili, l'anglais, le français? Pendant que tout ça était discuté dans la revue, les romanciers étaient à leur table de travail. Au jour de la décolonisation, il y avait déjà toute une bibliothèque affichant l'identité, les questionnements, les solutions, les problèmes de tout ce qui allait advenir à partir des indépendances.

Entre 1948 et 1960, il y a eu une abondance de livres, une véritable frénésie de publication. Dans cette période, Présence Africaine a publié environ la moitié de ceux qui sont devenus les classiques de la littérature africaine. Si on ajoute le Seuil, on arrive à quelque chose comme $70 \%$.

Il faut insister par ailleurs sur la liberté éditoriale de Présence. La maison publiait des controverses, comme l'ouvrage de Cheikh Anta Diop Nations nègres et cultures, que le milieu scientifique français trouvait ridicule. La lettre à Maurice de Thorez de Césaire, qui était vraiment une lettre, Alioune Diop a décidé de l'imprimer.

L'une des idées dominantes était celle de l'unité africaine. Alioune Diop savait que, malgré les différentes unions politiques, la religion, les traditions locales étaient importantes. Présence voulait donc aller au-delà des divisions, celles notamment qui avaient été imposées par les colonisateurs, comme la séparation entre anglophones et francophones.

\section{Présence Africaine a d'ailleurs influencé les anglophones...}

Il y a eu à un moment donné une édition anglaise de Présence, suivie d'une édition bilingue permanente. C'était une manière de réagir aux séparations imposées par la colonisation. Et c'est pourquoi, par exemple, le rôle de Présence Africaine a été si déterminant en Afrique du Sud, et si important au Nigeria. Si la revue Black Orpheus, créée par Ulli Beïer avec Wole Soyinka et quelques autres au Nigeria, a pris ce titre, c'est grâce à la préface de Sartre et à l'anthologie de Senghor, qui représentaient à leurs yeux le modèle de l'engagement du culturel au sein du politique. Modèle qui était relativement étranger dans les colonies anglophones, où l'action politique était essentiellement liée aux mouvements syndicaux.

En fait, dans le monde francophone, le discours sur la décolonisation a pu se faire grâce aux écrivains. On pourrait même dire grâce aux poètes. Dans une certaine mesure, cette prise de position des intellectuels dans le politique a également eu lieu dans les pays anglophones. Par exemple au Nigeria, dans les universités d'Ibadan ou d'Ifé, où le travail 
littéraire et culturel était lié à l'émancipation politique. Wole Soyinka et son théâtre en est l'exemple le plus frappant. Contrairement à ce que l'on pense et à la fameuse phrase de Wole Soyinka : "Le tigre ne proclame pas sa tigritude, mais il tue sa proie et la mange ", irrespectueusement adressée à Senghor, il n'y a jamais eu de conflit avec Présence. Soyinka a été longtemps à Paris et a beaucoup fréquenté Présence Africaine. Il était en lien très étroit avec Aimé Césaire et participait aux réflexions politiques et culturelles qui se faisaient sur l'avenir de l'Afrique et la décolonisation, et plus particulièrement sur le rôle de l'artiste et de l'homme de théâtre.

Plusieurs revues anglophones avaient des liens avec Présence Africaine : Africa South en Afrique du Sud (qui est devenu Africa South in Exile à Londres) ou encore Transition, l'importante revue de Rajat Neogy en Tanzanie. Il y avait des liens entre toutes ces revues alors que sur le plan politique chacun de ces mondes était hermétiquement clos, chacun avec son colonisateur. À cet égard, le Premier Congrès des écrivains et artistes noirs de 1956, qui avait amené des Nigériens, des Ghanéens, des Angolais, des Noirs américains et des Sud-Africains à Paris pour dialoguer avec leurs frères francophones, avait quelque chose de révolutionnaire. Cela paraît une évidence aujourd'hui, mais à l'époque cela ne s'était jamais vu. Cuba, les Antilles françaises sont aussi des sujets que l'on retrouve dans Présence Africaine. Par exemple, le comité pour l'autonomie des Antilles a été accueilli par Présence Africaine.

\section{Pouvez-vous nous parler du corpus littéraire créé par Présence Africaine?}

Présence Africaine était aussi en effet un lieu de réflexion sur la littérature. D’ailleurs, je trouve amusant de voir que plusieurs numéros de la revue ont été consacrés aux conditions d'une poésie et d'une littérature nationale africaine alors que parallèlement les auteurs publiaient des romans et de la poésie aux éditions. Comme la revue, les éditions publiaient sans idéologie particulière : des textes totalement réalistes, des textes autobiographiques, mais aussi Cahier d'un retour au pays natal, c'est-à-dire une modernité de langage, ou encore des poèmes d'agitation du jeune David Diop qui cherchait alors son esthétique (un poète mort trop jeune et qui n'a laissé qu'un unique recueil de poèmes, Coup de pilon), et en même temps la poésie de Jacques Rabemananjara, qui remontait à une tradition et la reproduisait dans une langue qu'on pourrait dire solennelle tout en étant un homme engagé. Rabemananjara a été emprisonné en 1947, il est sorti de prison au moment du congrès, en 1956, et entre-temps on publiait des textes de lui.

Présence Africaine publiait toutes les paroles. À aucun moment il n'y a eu d'esthétique collective, à aucun moment cela n'a été une école. Quand on voit la multiplicité des voies littéraires empruntées - poésie, roman, récit, essai -, la multiplicité des visions de l'histoire présentées - entre des gens comme Cheikh Anta Diop, qui remontait à l'origine nègre pour montrer la valeur de ces cultures, et d'autres auteurs qui se moquaient du passé, qui voulaient créer la modernité et pour qui les indépendances étaient une priorité -, c'était une gageure de réussir à faire le lien entre tous ces gens-là. Présence a joué là un rôle majeur.

\section{L'exposition présentée au musée du quai Branly s'achève au moment du Premier Festival des arts nègres, c'est-à-dire au moment des indépendances africaines. Que pensez-vous de cette césure?}

C'est le moment où l'épopée était terminée. L'heure des espérances réalisées et des tragédies nouvelles va prendre la suite. De 1948 jusqu'aux indépendances, il y a eu une véritable épopée. Elle avait même commencé pendant la guerre. On pense qu'il ne s'est rien passé pendant la guerre, mais à Paris il y avait des Noirs qui n'étaient pas pourchassés, qui faisaient des études et qui organisaient même des réunions pour parler de l'avenir de l'Afrique. Finalement, depuis les années 1930, au moment où tous ces intellectuels et artistes se sont connus, jusqu'à 1960, il y a eu une épopée collective à Paris. Pourquoi collective? 
On était tous dans le même combat, il fallait décoloniser tout le monde noir, tout le tiersmonde même. De plus, les politiques étaient sur place, les dizaines de députés africains qui étaient dans l'Union française et qui se battaient pour la décolonisation. Certains d'entre eux étaient même au gouvernement, comme Senghor, Houphouët-Boigny. C'était donc un mouvement collectif centré à Paris, qui était alors la capitale du tiers-monde francophone. Paris, c'est aussi le lieu où les Noirs américains ont rencontré pour la première fois des Africains, où les Antillais ont rencontré pour la première fois des Haïtiens, des Cubains. Paris était une capitale métisse depuis la fin du xıx siècle. Paris n'était pas uniquement la capitale de la France, c'était aussi une capitale de rencontres, de dialogue entre des gens qu'on avait éloignés les uns des autres, qu'on avait séparés. Cette rencontre n'était pas uniquement une rencontre de revendications, mais aussi de jouissance, de reconnaissance et de partage. En dépit du combat, il y avait un plaisir, une joie d'être ensemble. Présence Africaine n'avait rien de triste, et pourtant il y a eu des événements tristes, les massacres de Sétif dès 1945, ceux de Madagascar en 1947, ceux en Côte d'Ivoire en 1948... Au moment où Présence est apparue, les choses se présentaient très mal. Après la guerre et le mouvement libérateur commun avec les colonies, les Européens sont très vite redevenus les colonisateurs. On avait libéré le monde du fascisme ensemble, mais de nouveau on reprenait la colonisation. Évidemment, la résistance était très forte et, comme je le disais, cela a commencé par des massacres. Il y avait donc ce malheur dont il fallait venir à bout, mais il y avait aussi une jouissance. Nos ainés se vivaient libres, acteurs d'un grand recommencement du monde. Leur espérance était dans la droite ligne de l'espoir en l'Onu de 1947 : ils se disaient qu'ils allaient faire entrer les Noirs à l'Onu, les exclus à l'Unesco. À l'époque, à l'Unesco, il n'y avait que les pays indépendants. Je me souviens d'Alioune Diop montrant la carte de Bandung: sur la belle carte où il y avait tous les pays présents, il y avait un trou blanc qui était l'Afrique. Le congrès de 1956 est venu de là.

\section{Pouvez-vous nous expliquer l'importance historique de ce Premier Congrès des écrivains et artistes noirs, qui s'est tenu à la Sorbonne en septembre 1956 ?}

On n'avait pas de frontières, pas de pays, pas d'État, mais on était là. Nous étions là même si nous n'avions pas de statut politique, d'où ce congrès culturel et artistique de 1956 pour dire : "On est libres. " L'idée était de continuer le combat jusqu'à la libération politique. Qu'on ne vienne pas nous dire que nous étions silencieux, que nous n'existions pas et que nous étions un trou blanc sur la carte du monde. Alioune Diop voulait combler le trou blanc. Le congrès de 1956, c'était le désir de discuter ensemble parce qu'on savait très bien que les libérations allaient venir. On sentait le besoin de rester ensemble alors que le destin allait être de se séparer. On allait édifier des États, plus encore accoucher des nations, réunies en États-Unis d'Afrique. Il y avait donc ce désir de rassemblement, à la fois pour prendre des forces et dire qu'on n'allait pas se libérer seuls. Il fallait même appeler ceux qui étaient au-delà des frontières, c'est-à-dire les anglophones, les lusophones et les Noirs américains. Il y a d'ailleurs eu en 1956 un débat sur la situation des Noirs américains «colonisés de l'intérieur ». Ils se sont retrouvés embarqués dans la lutte anticoloniale avec cette phrase de Césaire : «Et nos frères américains eux-mêmes sont, par le jeu de la discrimination raciale, placés de manière artificielle et au sein d'une grande nation moderne, dans une situation qui ne se comprend que par référence à un colonialisme, certes aboli, mais dont les séquelles n'ont pas fini de retentir dans le présent. » Les Américains n'ont pas accepté cette comparaison, expliquant qu'ils avaient effectivement des problèmes mais qu'ils n'estimaient pas être des colonisés. Les débats de 1956 ont été très virulents autour de ce thème et ont caché les vrais engagements des uns et des autres. Le FBI était présent et les délégués américains ne pouvaient pas s'exprimer librement. Ils étaient attendus aux États-Unis par le 
maccarthysme. Il ne faut pas oublier qu'ils étaient sous surveillance. Malgré cela, il fallait qu'on se parle. Nos destins n'étaient pas les mêmes, le destin des Noirs américains n'était pas celui du Sénégal ou de la Guinée, ni celui de la Guadeloupe ou de Cuba ou encore d'Haïti. Il y avait tout de même cette idée centrale de se rencontrer. Ce sont les Noirs qui à ce moment-là étaient porteurs de cette universalité. Et si les Noirs ont décidé de se réunir, ce n'était pas face aux Blancs ou contre les Blancs. La salle était remplie de tous les amis avec lesquels ils travaillaient sur cette question. Je pense aux ethnologues, aux anthropologues, aux intellectuels et aux écrivains qui prenaient une part active à la décolonisation. Les nombreux messages le montrent bien. La salle n'était pas un «huis clos négriste». Il est important de rappeler une fois encore cette dimension d'ouverture. À ce moment-là, c'étaient les « Nègres " qui donnaient l'exemple de cette diversité culturelle, de cette ouverture au-delà des langues (le Congrès était bilingue français-anglais!), au-delà de l'histoire coloniale de chacun. Une autre chose qui réunissait tout le monde, c'était la soif de création et l'expression de nos identités distinctes. Plus on montrait qu'on était libres dans l'imaginaire et dans la création, plus on légitimait la nécessité de la libération politique à venir. Pour se libérer, il ne faut pas être prisonnier dans sa tête. La réalité est là. Ce qu'on appelle la francophonie, c'est-à-dire des gens qui se rencontrent dans un mouvement littéraire d'expression collective de leurs luttes, de leurs identités, date des anthologies de Damas (1947) et de Senghor (1948). La francophonie politique et institutionnelle est née des années plus tard. C'était la conséquence politique du fait que des gens avaient décidé de rester ensemble après les années 1960. 1960 a marqué la fin de l'épopée et le début des tragédies. Malgré cela, Présence Africaine défendait toujours l'idée selon laquelle il fallait garder le contact. D'ailleurs, au début des indépendances, l'idée d'union était toujours là. Senghor, par exemple, au début, était le président non du Sénégal, mais de la Fédération du Mali. On essayait de rester ensemble. Il y a eu le désir, sur le modèle des États-Unis et du début de l'Europe, de commencer à organiser les États-Unis d'Afrique. Et ça a été un grand échec, du fait de la manière dont les indépendances se sont faites. Nkrumah, président du Ghana, défendait l'idée du panafricanisme. Dans le monde de l'époque, avec les blocs qu'il y avait, c'est l'unité africaine qui devait faire le sort et le destin de l'Afrique. Alioune Diop défendait toujours l'idée que la liberté ne s'arrête pas avec la libération politique. La liberté était remise en cause par les libérateurs eux-mêmes. Il y a les exemples de Sékou Touré, des autres dictatures, mais aussi du néocolonialisme. Finalement, au moment des indépendances, le combat n'était pas terminé. On pensait que l'unité de l'Afrique pourrait se faire si on gardait des liens culturels et artistiques, d'où le Premier Festival mondial des arts nègres de Dakar en 1966. Tout le monde était dispersé, mais on voulait se réunir autour de la musique, du théâtre, de l'art... comme symboles vifs d'unité fondamentale et de diversité créatrice. Quand on y pense, cela paraît fou. L'Europe n'a jamais fait une chose pareille. L'Europe s'est d'abord construite sur des bases économiques. D'où l'importance de Présence, où le culturel n'était pas un cache-misère du politique. La culture était déjà une forme de liberté : à la fois le moyen et le but de l'émancipation.

\section{Pouvez-vous nous parler d'Alioune Diop et de l'importance historique de Présence Africaine?}

Alioune Diop était très discret. Il recevait les gens dans son bureau au 16, rue des Écoles, en mettant ses mains sur sa tête et en écoutant. Ce n'était pas quelqu'un qui se montrait sur les tribunes. Ce n'était pas un politique. En 1956, il a beaucoup œuvré pour que les politiques ne se mêlent pas du congrès. Senghor, qui était présent au congrès, était certes un homme politique, mais son intervention n'était pas politique. Cette vigilance était d'autant plus importante que les Européens colonisateurs voulaient préparer une décolonisation où l'Afrique serait toujours liée à l'Occident, cependant que les communistes, qui 
étaient les alliés de la décolonisation, envisageaient que les pays nouvellement indépendants rejoignent le bloc de l'Est. Il fallait donc tenir une position qui n'était pas de neutralité. Césaire exprime très bien la situation dans sa Lettre à Maurice Thorez : "Et c'est ici une véritable révolution copernicienne qu'il faut imposer, tant est enracinée en Europe, et dans tous les partis, et dans tous les domaines, de l'extrême droite à l'extrême gauche, l'habitude de faire pour nous, l'habitude de disposer pour nous, l'habitude de penser pour nous, bref l'habitude de nous contester ce droit à l'initiative dont je parlais tout à l'heure et qui est, en définitive, le droit à la personnalité. " Cette phrase résume très bien la posture qui a été celle d'Alioune Diop et du Présence de la grande époque. Ce n'était pas faute d'avoir des amis politiques qui étaient prêts à aider.

Pour finir, l'histoire de Présence Africaine appartient à celle du tiers-monde et à celle du monde, à la fois par l'influence qu'elle a eue ailleurs et par l'accueil qu'elle a réservé aux réflexions anthropologiques sur le développement qui valent pour l'ensemble des pays du tiers-monde. Toutes ces questions ont concerné le tiers-monde, comme celle-ci : quelle économie pour sortir de la dépendance coloniale? Tout ce qui précède montre que Présence était une revue, un mouvement, une éthique universalistes, portés par des gens qui étaient noirs au sens où ils ont fait là l'expérience de la lutte. C'étaient des gens qui étaient dans la modernité, pas seulement des gens qui étaient pour un retour à l'Afrique traditionnelle. Leur vision universaliste leur venait de l'expérience de l'abolition de l'esclavage, qui était une expérience universaliste même si c'étaient les Noirs qui étaient les esclaves.

La force de Présence tenait en grande partie aux personnalités qui la constituaient. Ou'est-ce que ces gens avaient de particulier pour pouvoir faire ça? Ils avaient une grande confiance en eux-mêmes, une grande confiance dans la personnalité de leur histoire, dans leur être et dans leur liberté. C'était une expérience de liberté à l'intérieur d'un monde aliéné, colonisé des deux côtés, en Afrique mais aussi en Europe. Dans une Europe colonisée par des idées qui ont abouti au fascisme, au nazisme... Là aussi, il y avait une pensée à libérer. Une liberté à créer collectivement et une création à libérer.

Propos recueillis par Sarah Frioux-Salgas 